An introduction to the theory of integration. By A. C. Zaanen. New York, Interscience, 1958. 9+254 pp. \$7.25.

In his preface to this graduate textbook Zaanen says, "I have sailed an intermediate course between the measure approach and the linear functional approach, fully realizing the danger that the attempt to do so may not find favour in the eyes of the extreme adherents of either school." Let me say at once that the attempt does find favor in my eyes. I would predict, however, that it will be better received by the measure school than by the linear functional school. Indeed, I would classify it informally as "the Daniell integral for measure theorists." As such I think it is a welcome addition to the literature.

There naturally arises the "hen or egg" question of whether Zaanen gets the integral from a measure or vice versa. Briefly, his procedure is as follows. He starts with a measure (non-negative, countably additive set function) on a semi-ring and extends it via straight Caratheodory theory of exterior measure to a measure on a $\sigma$-ring. $\mathrm{He}$ then takes a linear lattice of non-negative point functions on an abstract space and defines an integral as a monotone, linear functional on this lattice which is continuous at zero with respect to monotone convergence. Next, he introduces the ordinate sets for these point functions and shows that they form a semi-ring. The integral on the lattice of point functions induces a measure on the semi-ring of ordinate sets. Extension of this measure to a $\sigma$-ring of ordinate sets generates the non-negative, measurable functions and their integrals. The only delicate problem is preservation of linearity for the extended integral. For a point function with variable sign the integral is defined as usual. When all the shouting is over we have as a theorem that the summable functions form a complete metric space in which the original linear lattice is a dense set.

By way of motivating this discussion, Zaanen turns at every oppotunity to the example of Lebesgue measure and Lebesgue integral. The half-open intervals form a prime example of a semi-ring, and the step functions with their integrals defined in the obvious way furnish an example through which the linear lattice with Daniell integral is introduced.

A Stieltjes-Lebesgue integral is defined as one generated by a lattice of step functions on a measure space, and a chapter is devoted to the study of these-including the case of complex integrands. After this follows the Fubini theorem, $L_{p}$ spaces, the Radon-Nikodym theorem, and differentiation of set functions. In line with Zaanen's announced intention of designing a course that can be given in a limited number of lecture hours, all this is completed on p. 157. 
The remainder of the book consists of "optional" topics, the major ones being change of variable in an integral, signed and complex measures, conjugate spaces and weak convergence, Fourier transforms, and ergodic theory.

The book is well supplied with exercises and should make quite a satisfactory text. Its brevity comes not from terseness but from judicious editing. I would guess that students will find it quite readable and that professors will object because their pet theorems are missing.

I have three specific criticisms.

(i) Zaanen defines $I(f)$ - the integral of $f$-as $I\left(f^{+}\right)+I\left(f^{-}\right)$. He calls $f$ integrable if this exists and summable if it is finite. I object mildly to this terminology, but my real criticism is that he has listed $\infty-\infty=0$ as a postulate for the extended real number system. So, I do not think he means what he says about integrability.

(ii) He does not define the word function and seems to use $f$ and $f(x)$ interchangeably. In certain transfinite proofs he refers quite properly to a "union of functions" with a parenthetic explanation that will probably be meaningless to the student who has never seen a function defined as a set of ordered pairs.

(iii) $\mathrm{He}$ introduces the metric space of measurable sets with the measure of the symmetric difference as a distance function but so far as I can discover he does not prove it complete or even discuss the question of completeness. Yet, on p. 183 he applies the Baire category theorem to this space deriving the usual inference, and this requires completeness.

\section{E. MunRoe}

Introduction to difference equations. By Samuel Goldberg. New York, Wiley, 1958. $12+260$ pp. $\$ 6.75$.

This extremely well-written book is "primarily intended for social scientists who wish to understand the basic ideas and techniques involved in setting up and solving difference equations." In the introduction, examples are given of problems which give rise to difference equations-a learning model and the study of the variations in national income. Throughout, numerous exercises are chosen from economics, psychology, and sociology with references to the literature of these subjects. Discussions are devoted to economic dynamics, inventory analysis, learning models, communication theory, and income variation.

On the mathematical side, the author gives an extremely lucid and careful explanation of the techniques of solving linear difference equa- 